\title{
Exposed Population
}

National Cancer Institute

\section{Source}

National Cancer Institute. Exposed Population. NCl Thesaurus. Code C71551.

The members of a study who have received the treatment. 